\title{
Avaliação Nutricional de Pacientes com Insuficiência Renal Crônica Submetidos à Hemodiálise em uma Clínica de Nefrologia em João Pessoa-PB
}

\author{
Nutritional Assessment of Patients with Chronic Renal Failure Undergoing \\ Hemodialysis in a nephrology clinic in João Pessoa-PB
}

\author{
ARABELA VIEIRA CLEMENTINO' \\ ALESSANDRA DE FÁTIMA OLIVEIRA PATRÍCIO' \\ PAMELA RODRIGUES MARTINS LINS ${ }^{2}$ \\ SÔNICA CRISTINA PEREIRA DE OLIVEIRA ${ }^{2}$ \\ MARIA DA CONCEIÇÃO RODRIGUES GONÇALVES ${ }^{3}$
}

\section{RESUMO}

Objetivo: Caracterizar o estado nutricional dos pacientes com Insuficiência Renal Crônica submetidos à hemodiálise de uma Clínica de Nefrologia em João Pessoa- PB. Material e Métodos: A amostra foi composta por 43 pacientes, de ambos os sexos, submetidos à hemodiálise. Para coleta dos dados foi utilizada uma ficha clínica com dados socioeconômicos; informações sobre a doença; dados antropométricos e bioquímicos. Para análise dos resultados foi realizado o teste de associações pelo Qui quadrado, utilizando o software SPSS 21. Resultados: Observou-se que $56 \%$ dos pacientes eram do sexo masculino, com idade média de 39,79 $\pm 11,42$ anos, $23 \%$ dos pacientes avaliados tinham o ensino fundamental completo, e a maioria, $37 \%$, referiam receber um salário mínimo. Quanto à etiologia da Insuficiência Renal Crônica à Glomerulonefrite crônica era a doença mais prevalente em ambos os sexos. Identificou-se que $63 \%$ das mulheres apresentaram-se eutróficas segundo o Índice de Massa Corporal. Com relação à Circunferência do Braço, Circunferência Muscular do Braço e Área Muscular do Braço corrigida os homens foram classificados com desnutrição, revelando um déficit de massa muscular. Em relação aos indicadores bioquímicos apenas a ureia e o cálcio apresentaram médias não aceitáveis em ambos os sexos. Conclusões: A maioria dos homens avaliados apresentou déficit de massa muscular, enquanto a maioria das mulheres encontravam-se eutróficas de acordo com os mesmos parâmetros utilizados. No presente estudo evidenciamos que não existe um parâmetro que forneça de forma completa o estado nutricional, sendo necessário utilizar diversos indicadores em conjunto que permitam identificar riscos ou anormalidades nutricionais já instalados.

\section{DESCRITORES}

Insuficiência Renal. Estado Nutricional. Hemodiálise.

\begin{abstract}
Objective: To characterize the nutritional status of patients with chronic renal failure undergoing hemodialysis in a nephrology clinic in João Pessoa, PB, Brazil. Material and Methods: The sample consisted of 43 patients of both sexes undergoing hemodialysis. A case report form with socioeconomic profile, information about the disease, and anthropometric and biochemical data, was used for data collection. The data were analyzed on SPSS 21 statistical package using the Chi square test. Results: $56 \%$ of patients were male, with a mean age of $39.79 \pm 11.42$ years; $23 \%$ of the patients had completed elementary school, and the majority $(37 \%)$ reported an income of one minimum wage. As to the etiology of chronic kidney disease, chronic glomerulonephritis was the most prevalent condition in both sexes. A total of $63 \%$ of women were eutrophic based on their Body Mass Index values. With regard to the arm circumference, arm muscle circumference and corrected arm muscle area, men were classified as having malnutrition revealing a muscle mass deficit. Among the biochemical indicators, only urea and calcium were exceeding the acceptable reference means in both sexes. Conclusions: The majority of the men investigated were found to have muscle deficit, while most women had normal weight based on the same parameters. In this study we showed that there is not a single parameter to comprehensively elucidate the nutritional status of the individual, making it necessary to use several indicators to identify existing risks or nutritional abnormalities.
\end{abstract}

\section{DESCRIPTORS}

Renal Insufficiency. Nutritional Status. Hemodialysis.

\footnotetext{
Graduada pela Universidade Federal da Paraíba (UFPB), João Pessoa, PB - Brasil.

Professora Mestre do Departamento de Nutrição da Universidade Federal da Paraíba (UFPB), João Pessoa/PB, Brasil.

Professora Doutora do Departamento de Nutrição da Universidade Federal da Paraíba (UFPB), João Pessoa/PB, Brasil.
} 
A doença renal crônica tem sido considerada um importante problema de saúde pública. Atualmente a Insuficiência Renal Crônica tem sido definida como um termo genérico de desordem heterogênica que afeta a estrutura e a função dos rins. Assim a definição é baseada em três componentes: (1) comportamento anatômico ou estrutural; (2) um componente funcional, baseada na taxa de filtração glomerular; e (3) um componente temporal ${ }^{1}$.

No Brasil, dados do Censo 2013 da Sociedade Brasileira de Nefrologia revelam que 100.397 pacientes são submetidos a tratamento dialítico, sendo que $25 \%$ encontram-se na Região Nordeste. A grande maioria destes pacientes $(90,7 \%)$ é submetida à hemodiálise como terapia renal substitutiva. Apesar dos benefícios da hemodiálise, que permitem prolongar a vida dos pacientes com doenças renais crônicas, as condições impostas pela doença e pelo próprio tratamento dialítico resultam em uma série de alterações orgânicas, com complicações agudas e crônicas, e nutricionais ${ }^{2}$.

Esforços vêm sendo realizados no sentido de melhor compreender os fatores envolvidos na condição nutricional desses pacientes e contribuir não somente para a redução das taxas de mortalidade, mas também para a melhoria da qualidade de vida desses enfermos ${ }^{3}$.

A Insuficiência renal crônica pode ser tratada inicialmente por meio de medidas terapêuticas conservadoras, como: tratamento dietético, medicamentoso e controle da pressão arterial. A indicação do programa dialítico será feita quando o tratamento conservador não é capaz de manter a qualidade de vida do paciente renal e quando há o surgimento de sinais e sintomas importantes de uremia ${ }^{4}$.

A causa da desnutrição é multifatorial e inclui ingestão alimentar deficiente, distúrbios hormonais e gastrointestinais, restrições dietéticas, uso de medicamentos que podem influenciar na absorção de nutrientes, diálise insuficiente e presença constante de enfermidades associadas. Além disso, a uremia, a acidose metabólica procedimento de hemodiálise, por si só, são hipercatabólicos e estão associados à presença de estado inflamatório A caracterização do estado nutricional desses enfermos é importante tanto para prevenir a má nutrição quanto para indicar adequada intervenção nutricional nos desnutridos submetidos à diálise ${ }^{5}$.

Avaliar e caracterizar o perfil nutricional dos pacientes com Insuficiência Renal Crônica submetidos à hemodiálise é fundamental para a prevenção e tratamento dos distúrbios nutricionais, no qual se deve acompanhar periodicamente o estado nutricional destes pacientes para reduzir o risco de infecções e outras complicações.
Neste contexto a pesquisa teve como objetivo geral caracterizar o estado nutricional dos pacientes com insuficiência renal crônica submetidos à hemodiálise de uma clínica de nefrologia em João Pessoa- PB.

\section{MATERIAL E MÉTODOS}

Constitui-se de um estudo descritivo e transversal, com abordagem quantitativa e amostragem por conveniência, sendo composta por 43 pacientes com insuficiência renal crônica que são submetidos ao tratamento de hemodiálise em uma clínica de nefrologia em João Pessoa- PB, a partir da demanda espontânea do serviço durante o período de agosto a dezembro de 2013, que concordaram em assinar o Termo de Consentimento Livre e Esclarecido - TCLE. O projeto para esse estudo foi submetido e aprovado pelo Comitê de Ética do Centro de Ciências da Saúde da Universidade Federal da Paraíba, sendo o número do protocolo 0238/ 13.

Foram incluídos na pesquisa pacientes com idade de 20 - 59 anos considerados adultos segundo a Organização Mundial de Saúde, com Insuficiência Renal Crônica em programa de hemodiálise, sem intercorrências clínicas ou interrupção no tratamento e em condições plenas de comunicar-se e informar o estado de saúde. Foram excluídos os pacientes amputados, os hospitalizados, com doenças inflamatórias, infecções recentes e insucessos de transplante renal nos últimos seis meses.

Os dados foram coletados na própria Clínica de Nefrologia a partir de uma ficha clínica. Nesta, foram anotados os dados socioeconômicos como a idade, sexo, renda e escolaridade; informações sobre a doença como etiologia e tempo de tratamento; dados antropométricos e os dados bioquímicos.

A avaliação antropométrica foi realizada após a sessão de hemodiálise, quando estavam próximo ao peso seco. Foram obtidos peso e altura utilizando balança mecânica Filizola de capacidade até $150 \mathrm{~kg}$ com estadiômetro fixado. A circunferência do braço (CB) foi medida em centímetros utilizando-se fita inelástica e a dobra cutânea tricipital (DCT) em milímetros utilizando o adipômetro (Cescof). As aferições foram realizadas no braço oposto ao da fístula arteriovenosa, sendo DCT aferida em triplicata para a obtenção da média.

A partir das medidas aferidas foram calculados os seguintes parâmetros: índice de Massa Corporal (IMC), circunferência muscular do braço (CMB), área muscular do braço corrigida (AMBc), adequação de DCT (\%DCT) e adequação de CB (\%CB).

O IMC foi calculado por meio da razão do peso 
corporal e o quadrado da altura, sendo classificado segundo a Organização Mundial da Saúde ${ }^{6}$.

A CMB foi calculada a partir da seguinte fórmula: $\mathrm{CMB}(\mathrm{cm})=\mathrm{CB}(\mathrm{cm})-$ ð $[\mathrm{PCT}(\mathrm{mm}) \div 10]$. Os resultados obtidos da DCT, CB e CMB foram comparados aos valores propostos por Frisancho ${ }^{7}$ e posteriormente efetuadas as adequações e classificadas de acordo com Blackburn; Thornton ${ }^{8}$, sendo considerado dentro dos parâmetros da normalidade valores entre 90 - $110 \%$.

$\mathrm{AAMBc}$ foi calculada pelas fórmulas: Homens: $\operatorname{AMBc}\left(\mathrm{cm}^{2}\right)=[\mathrm{CB}(\mathrm{cm})-\text { ð } \mathrm{PCT}(\mathrm{mm}) \div 10]^{2}-10 \div 4 \mathrm{x}$ ð. Mulheres: $\mathrm{AMBc}\left(\mathrm{cm}^{2}\right)=[\mathrm{CB}(\mathrm{cm})-$ ð $\mathrm{x}$ PCT $(\mathrm{mm}) \div$ $10]^{2}-6,5 \div 4$ x ð e comparadas a tabela de percentil de Frisancho $^{7}$, sendo o estado nutricional classificado de acordo com Riella; Martins ${ }^{9}$.

A avaliação bioquímica foi utilizada para determinar o estado nutricional e a função renal dos pacientes submetidos à hemodiálise a partir dos níveis séricos de albumina, uréia, creatinina, potássio, cálcio e fósforo. Os resultados dos exames foram obtidos por meio dos prontuários de cada paciente, com a data mais próxima da avaliação antropométrica. O estado nutricional a partir dos valores de albumina foi classificado de acordo com Martins ${ }^{10}$ e os valores dos demais indicadores bioquímicos foram classificados de acordo com Martins; Cardozo ${ }^{11}$.

Os dados referentes à ficha clínica foram analisados no Microsoft Office Excel® 2010, com propósito de mensurar a frequência absoluta (n), e a frequência relativa $(\%)$, sendo realizada a média e desvio padrão para indicar a variabilidade dos dados. Foi realizado também o Teste de associações pelo Qui quadrado, utilizando o software SPSS 21.

\section{RESULTADOS}

O estudo envolveu uma amostra de 43 pacientes portadores de Insuficiência Renal Crônica submetidos à hemodiálise, distribuídos em $24(56 \%)$ homens com média de 39,79 $\pm 11,42$ anos de idade e $19(44 \%)$ mulheres com média de 38,53 $\pm 11,47$ anos de idade, (Tabela 1).

Foi realizado o teste de Qui quadrado associando a escolaridade dos pacientes avaliados com a classificação do índice massa corporal e a classificação da circunferência muscular do braço, no qual não foi observada diferença estatística, obtendo-se respectivamente $\mathrm{p}=$ 0,846 e $\mathrm{p}=0,566$.

$\mathrm{Na}$ associação feita pelo teste do Qui quadrado não foi verificado diferença estatística entre a renda e classificação do IMC, sendo obtido um $\mathrm{p}=0,852$.

Um fator relevante para este estudo é a média do tempo de tratamento que foi maior nos homens quando comparado ao das mulheres (Tabela 2).

$\mathrm{Na}$ amostra de mulheres foram identificadas 7 patologias que correspondia a etiologia da IRC, como podemos observar no Figura 1.

\begin{tabular}{|c|c|c|}
\hline Sexo & $\mathrm{N}$ & $\%$ \\
\hline Feminino & 19 & 44 \\
\hline Masculino & 24 & 56 \\
\hline \multicolumn{3}{|l|}{ Idade } \\
\hline $21-40$ anos & 23 & 53.6 \\
\hline 41.59 anos & 20 & 46,4 \\
\hline \multicolumn{3}{|l|}{ Grau de Instrucão } \\
\hline Ens. Fund. Completo & 10 & 23 \\
\hline Ens. Fund. Incompleto & 5 & 12 \\
\hline Ens. Méd. completo & 5 & 12 \\
\hline Ens. Méd. Incompleto & 10 & 23 \\
\hline Ensino Superior & 4 & 9 \\
\hline Analfabeto & 9 & 21 \\
\hline \multicolumn{3}{|l|}{ Renda Familiar } \\
\hline 1 salário minimo & 16 & 37 \\
\hline 2 salários minimos & 12 & 28 \\
\hline 3 ou mais salários minimos & 15 & 35 \\
\hline
\end{tabular}


Tabela 2. Média do tempo de tratamento hemodialitico das mulheres e dos homens portadores de Insuficiência Renal Crónica de uma clinica de nefrologia em Joāo Pessoa no ano de 2013.

Sexo Tempo de tratamento Desvio padrão

$\begin{array}{lll}\text { Feminino } & 48,79 & \pm 59,61\end{array}$

Masculino $\quad 88,19 \quad \pm 73,52$

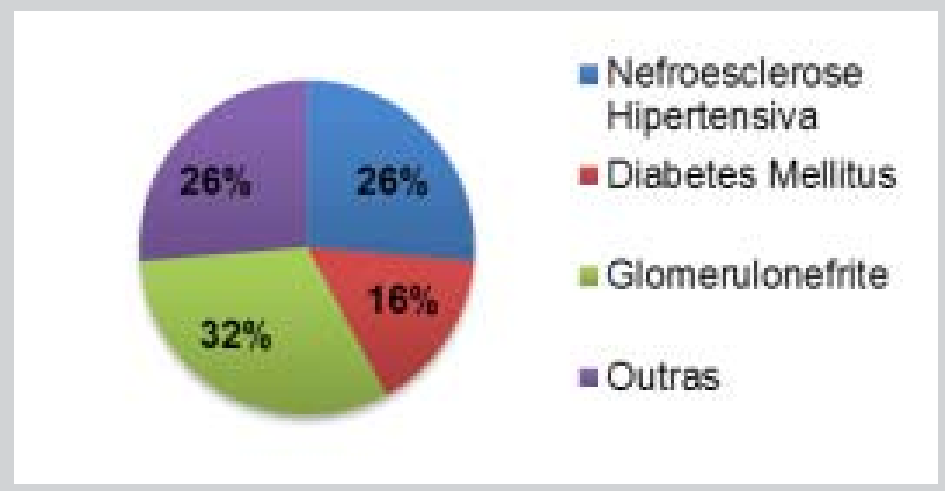

Figura 1. Prevalência da etiologia da Insuficiência Renal Crônica nas mulheres submetidas à hemodiálise de uma clínica de nefrologia em João Pessoa.

Como demonstra a Figura 2, na amostra dos homens foram identificadas 4 patologias referentes à doença de base dos pacientes.

Quanto ao teste de associação do Qui quadrado da amostra dos 43 pacientes avaliados não foi observado diferença estatística entre a etiologia da IRC e os valores de classificação como aceitável e não aceitável da ureia, obtendo-se $\mathrm{p}=0,397$.
Com base na média do IMC a maioria dos pacientes avaliados tanto do sexo masculino $(24,18 \mathrm{~kg} /$ $\left.\mathrm{m}^{2} \pm 5,76 \mathrm{~kg} / \mathrm{m}^{2}\right)$ como do sexo feminino $(22,38 \pm 4,09 \mathrm{~kg} /$ $\mathrm{m}^{2}$ ) apresentaram dentro do limite da normalidade, com predomínio dos pacientes eutróficos seguidos daqueles com desnutrição e sobrepeso (Tabela 3).

No teste do Qui quadrado associando a classificação do IMC com classificação da CMB e com

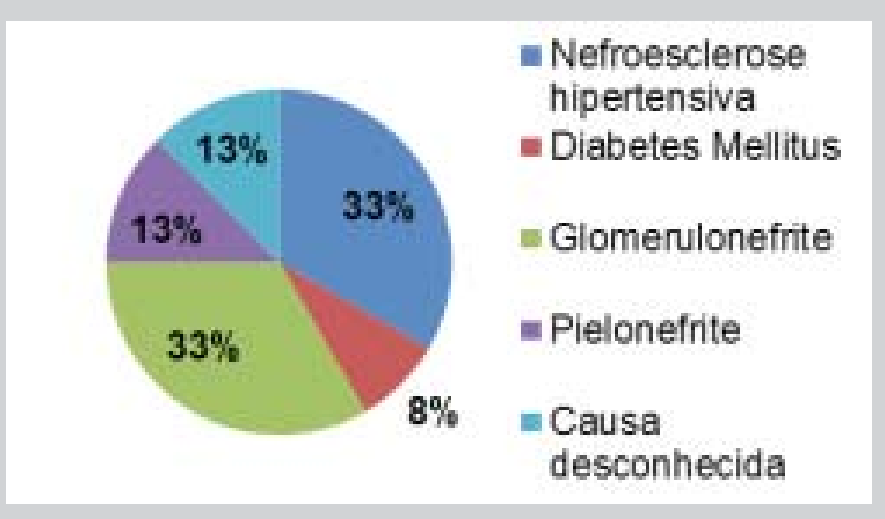

Figura 2. Prevalência da etiologia da Insuficiência Renal Crônica nos homens submetidos à hemodiálise de uma clínica de nefrologia em João Pessoa. 


\begin{tabular}{|c|c|c|c|c|}
\hline Estado Nutricional de 20-59 Anos & $\mathrm{Mu}$ & eres & & \\
\hline & $\mathrm{N}$ & $\%$ & $\mathrm{~N}$ & $\%$ \\
\hline \multicolumn{5}{|l|}{ IMC } \\
\hline Desnutricão & 3 & 16 & 4 & 17 \\
\hline Eutrofia & 12 & 63 & 13 & 54 \\
\hline Sobredeso & 3 & 16 & 3 & 13 \\
\hline Obesidade Grau I & 1 & 5 & 2 & 8 \\
\hline Obesidade Grau II & 0 & 0 & 2 & 8 \\
\hline
\end{tabular}

o valor aceitável e não aceitável de creatinina não foi verificado diferença estatística, no qual essas associações apresentaram respectivamente o valor de $\mathrm{p}=0,108$ e $\mathrm{p}=0,742$

A maioria dos pacientes avaliados de ambos os sexos não apresentaram depleção de gordura corporal através da PCT, como podemos evidenciar na Tabela 4.

De acordo os valores de adequação da circunferência do braço que é um indicador antropométrico que avalia a massa muscular e o tecido adiposo podemos observar na Tabela 4 que os homens obtiveram um percentual maior de desnutrição leve (29\%), enquanto as mulheres obtiveram um percentual superior para eutrofia $(63 \%)$.

De acordo com a adequação da circunferência muscular do braço que é uma medida de avaliação do compartimento protéico somático e da média da área muscular do braço corrigida que avalia a reserva de tecido muscular corrigindo a área óssea, podemos perceber na Tabela 4 que os pacientes do sexo masculino apresentaram 34\% com desnutrição moderada segundo a CMB e 54\% com desnutrição grave segundo a AMBc. Enquanto as mulheres apresentaram maior percentual de eutrofia (74\%) de acordo com AMBc os homens apresentaram percentual superior de desnutrição grave (54\%).

Os valores de albumina obtidos a partir dos exames bioquímicos foi utilizado como critério laboratorial de reserva proteica para classificação do estado nutricional dos pacientes em hemodiálise.

Os valores de albumina das mulheres revelaram que $58 \%$ destas apresentavam desnutrição leve com valores entre 3,1-3,9 mg/dl, sendo que $45 \%$ estavam dentro dos valores considerados adequados (e" $4 \mathrm{mg}$ / dl). As mulheres obtiveram média do valor de albumina de 3,73 $\pm 0,36 \mathrm{mg} / \mathrm{dl}$, (Tabela 5).

Quanto aos homens, $63 \%$ destes apresentaram dentro do valor adequado e" $4 \mathrm{mg} / \mathrm{dl}$, no qual $33 \%$ foi classificado com desnutrição leve e $4 \%$ com desnutrição moderada apresentando valor de albumina entre 2,1$3 \mathrm{mg} / \mathrm{dl}$. A média do valor de albumina dos homens foi semelhante à média obtida das mulheres sendo de 3,8 $\mathrm{mg} / \mathrm{dl} \pm 0,51$.

Como podemos observar na Tabela 6 os níveis de ureia e cálcio das mulheres e dos homens apresentaram valores abaixo do recomendado, enquanto os níveis de creatinina, fósforo e potássio estão dentro dos valores aceitáveis para os pacientes renais.

Analisando a associação dos indicadores bioquímicos (ureia e creatinina) com o IMC, não foi observada diferença estatística, sendo os valores respectivamente de $\mathrm{p}=0,99$ e $\mathrm{p}=0,74$.

\section{DISCUSSÃO}

De acordo com o que se pôde observar no estudo, houve um predomínio da participação dos indivíduos do sexo masculino, representando a maioria da amostra, assim corroborando com os resultados de duas pesquisas ${ }^{12-13}$, no qual ambos os autores avaliaram o estado nutricional dos pacientes com IRC submetidos à hemodiálise. Estes resultados sugerem que o sexo masculino torna-se mais suscetível à doença do que o sexo feminino, uma vez que os homens procuram menos os serviços de saúde do que as mulheres ${ }^{14}$.

Em relação à faixa etária dos pacientes avaliados, os homens e as mulheres apresentaram média de idade semelhante, o que também mostra em outro estudo ${ }^{15}$ que avaliou 17 pacientes com predominância do sexo masculino com IRC em duas Unidade de Hemodiálise, no qual a idade das mulheres variavam entre $18-57$ anos com média de $35 \pm 15$ anos de idade e de homens entre 19 - 59 anos de idade com média de $38 \pm 17$ anos. 


\begin{tabular}{|c|c|c|c|c|}
\hline \multicolumn{5}{|c|}{$\begin{array}{l}\text { Tabela 4. Classificação Nutricional segundo } \\
\text { a Adequação da Dobra Cutānea Tricipital, } \\
\text { Circunferència do Braço, Circunferência } \\
\text { Muscular do Braço e da Área Muscular do } \\
\text { Braço corrigida das Mulheres e Homens com } \\
\text { Insuficiência Renal Crônica submetidas à } \\
\text { Hemodíálise de uma Clinica de Nefrologia } \\
\text { em Joâo Pessoa. no ano de } 2013 \text {. }\end{array}$} \\
\hline \multirow{2}{*}{$\begin{array}{l}\text { Estado } \\
\text { Nutricional }\end{array}$} & \multicolumn{2}{|c|}{ Mulheres } & \multicolumn{2}{|c|}{ Homens } \\
\hline & $\mathrm{N}$ & $\%$ & $\mathrm{~N}$ & $\%$ \\
\hline \multicolumn{5}{|l|}{ DCT } \\
\hline Desnutricão qrave & 4 & 21 & 3 & 12 \\
\hline $\begin{array}{l}\text { Desnutrição } \\
\text { moderada }\end{array}$ & 4 & 21 & 3 & 12 \\
\hline Desnutrição leve & 5 & 26 & 4 & 17 \\
\hline Eutrofia & 6 & 32 & 10 & 42 \\
\hline Obesidade & 0 & 0 & 4 & 17 \\
\hline \multicolumn{5}{|l|}{ CB } \\
\hline Desnutricão arave & 1 & 5 & 3 & 13 \\
\hline $\begin{array}{l}\text { Desnutrição } \\
\text { moderada }\end{array}$ & 1 & 5 & 6 & 2 \\
\hline Desnutricão leve & 5 & 27 & 7 & 29 \\
\hline Eutrofia & 12 & 63 & 6 & 25 \\
\hline Sobrepeso & 0 & 0 & 1 & 4 \\
\hline Obesidade & 0 & 0 & 1 & 4 \\
\hline \multicolumn{5}{|l|}{ CMB } \\
\hline Desnutrição grave & 1 & 5 & 2 & 8 \\
\hline $\begin{array}{l}\text { Desnutriçăo } \\
\text { moderada }\end{array}$ & 0 & 0 & 8 & 34 \\
\hline Desnutriçăo leve & 3 & 16 & 6 & 25 \\
\hline Eutrofia & 14 & 74 & 6 & 25 \\
\hline Sobrepeso & 1 & 5 & 2 & 8 \\
\hline \multicolumn{5}{|l|}{$\mathrm{AMBC}$} \\
\hline Desnutrição qrave & 2 & 10 & 13 & 54 \\
\hline $\begin{array}{l}\text { Desnutriçắo } \\
\text { leveimoderada }\end{array}$ & 3 & 16 & 4 & 17 \\
\hline Eutrofia & 14 & 74 & 7 & 29 \\
\hline
\end{tabular}

Tabela 5. Classificação Nutricional da amostra por gênero a partir da reserva da Albumina das mulheres e dos homens com Insuficiência Renal Crônica submetidas à hemodiálise de uma clínica de nefrologia em Joāo Pessoa, no ano de 2013.

\begin{tabular}{lcccccc}
$\begin{array}{l}\text { Classificaçāo Nutricional } \\
\text { (Albumina) }\end{array}$ & \multicolumn{2}{c}{ Feminino } & \multicolumn{3}{c}{ Masculino } & \multicolumn{2}{c}{ Total } \\
\hline & N & $\%$ & N & $\%$ & N & $\%$ \\
\hline Adequado & 8 & 42 & 15 & 63 & 23 & 54 \\
Desnutricăo leve & 11 & 58 & 8 & 33 & 19 & 44 \\
Desnutricăo moderada & - & - & 1 & 4 & 1 & 2 \\
Total geral & 19 & 100 & 24 & 100 & 43 & 100 \\
\hline
\end{tabular}




\begin{tabular}{|c|c|c|c|}
\hline $\begin{array}{c}\text { Exames } \\
\text { Bioquímicos }\end{array}$ & Feminino & Masculino & \\
\hline & $\begin{array}{c}\text { Média } \\
\text { Desvio } \pm \text { Padrão }\end{array}$ & $\begin{array}{c}\text { Média } \\
\text { DesviotPadrão }\end{array}$ & $\begin{array}{c}\text { Valor } \\
\text { Aceitável }\end{array}$ \\
\hline Uréia( maidl) & $129,15 \pm 28,42$ & $128,5 \pm 39,24$ & $130-200 \mathrm{ma} / \mathrm{dL}$ \\
\hline Creatinina( ma/d) & $9,19 \pm 2,39$ & $10,06 \pm 3,98$ & $7-12 \mathrm{ma} / \mathrm{dl}$ \\
\hline Cálcio (maldl) & $8.9 \pm 0.76$ & $8,42 \pm 0,86$ & $9-11 \mathrm{ma} / \mathrm{dl}$ \\
\hline Fósforo (ma/dl) & $5,08 \pm 0,99$ & $5,10 \pm 0,9$ & $4,5-6 \mathrm{mg} / \mathrm{dl}$ \\
\hline Potássio (moidl) & $5,4 \pm 1,34$ & $5,5 \pm 0,74$ & $3,5-5,5 \mathrm{mg} / \mathrm{dl}$ \\
\hline
\end{tabular}

Quanto ao grau de instrução os achados obtidos no presente estudo diferem dos resultados obtidos em um pesquisa ${ }^{16}$, em que ao analisarem as características dos pacientes renais crônicos submetidos a tratamento hemodialítico segundo variáveis sócio-demográficas verificaram percentuais menores de pacientes analfabetos e com ensino médio incompleto, tendo a maioria destes ensino médio completo.

As informações sobre o grau de escolaridade dos pacientes podem ser utilizadas como um instrumento no auxílio à comunicação, ou seja, são indicadores de alerta para que os profissionais de saúde utilizem uma linguagem compatível que permita um entendimento ideal, no que diz respeito à orientação nutricional e clínica, educação e prevenção de complicações decorrentes do próprio tratamento ${ }^{17}$.

Segundo a renda familiar, o percentual dos pacientes que possuía um salário mínimo foi semelhante ao que possuía 3 ou mais SM, sendo esse resultado divergente ao verificado no estudo de outros autores ${ }^{18}$ que apresentou maior percentual $(71 \%)$ de pacientes que possuíam de um a dois SM.

Outros autores ${ }^{19}$ ao avaliar 575 pacientes com IRC submetidos à hemodiálise demonstrou a partir do teste de Kappa que os indivíduos com renda inferior ou igual a um SM tiveram 1,6 a 4,2 vezes mais probabilidade de ter desnutrição do que aqueles com renda maior que um SM, obtendo um valor de $\mathrm{p}<0,05$ (significativo), no qual no presente estudo a partir do teste do Qui quadrado não foi verificado diferença estatística quanto à associação da renda e a classificação do IMC $(\mathrm{p}=0,852)$.

Outro fator relevante para este estudo é média do tempo de tratamento que foi maior nos homens quando comparado ao das mulheres. Em um estudo ${ }^{20}$ ao entrevistarem 40 pacientes renais crônicos sob tratamento hemodialítico com idade superior a 18 anos, verificou também maior tempo de tratamento para os homens, com média de 40,9 meses, no qual segundo o autor não há justificativas plausíveis para tal fenômeno. Esses resultados não foram semelhantes à pesquisa ${ }^{14}$ que evidenciou tempo de tratamento semelhante entre homens e mulheres.

O Censo da Sociedade Brasileira de Nefrologia ${ }^{21}$ estabeleceu como principal causa da insuficiência renal a hipertensão arterial sistêmica (35\%), seguido da diabetes mellitus (30\%) e glomerulonefrite crônica (12\%). No presente estudo, as patologias que mais prevaleceram como fator causal da IRC corroboram com os resultados de outras pesquisas ${ }^{12-22}$ que apresentaram como principais doenças de base a glomerulonefrite crônica, nefroesclerose hipertensiva e diabetes mellitus. Esses dados diferem em partes dos resultados dos autore ${ }^{14}$ que demostraram maior frequência da doença policística como causa da IRC.

$\mathrm{Na}$ literatura, mais de $30 \%$ dos pacientes que iniciam diálise são diabéticos. A morbidade e mortalidade são substancialmente maiores em pacientes diabéticos do que nos demais pacientes não diabéticos, sendo as doenças cardiovasculares e as infecções as principais causas de morte 4 .

Em relação à antropometria realizada, o IMC é um dos indicadores mais utilizados na prática clínica, por causa da estreita associação com a morbimortalidade da população em diversas condições clínicas. Nos pacientes com IRC é recomendável que a interpretação do IMC seja feita em associação com outros marcadores nutricionais, pois seus valores podem ficar "mascarados" em situações de retenção hídrica ${ }^{23}$.

Com base na média do IMC a maioria dos pacientes avaliados tanto do sexo masculino como sexo feminino apresentaram dentro do limite da normalidade, bem como a média encontrada nos resultados de outra pesquisa ${ }^{24}$ que foi de $24,28 \pm 4,38 \mathrm{~kg} / \mathrm{m}^{2}$ para as mulheres e de $24,62 \pm 4,51 \mathrm{~kg} / \mathrm{m}^{2}$ para os homens, realizado com 
148 indivíduos com IRC mantidos em hemodiálise com idade média de $51 \pm 15,63$ anos, no qual destes $60,14 \%$ eram do sexo masculino corroborando com o presente estudo.

Assim como os resultados de um trabalho realizado anteriormente ${ }^{12}$ que observou que $68,5 \%$ dos pacientes em tratamento hemodiálitco apresentaram eutróficos, 15,9 \% com desnutrição e sobrepeso para ambos os sexos. Portanto diferem dos dados encontrados em uma pesquisa ${ }^{25}$ que utilizou parâmetros de classificação do IMC diferente ao presente estudo. Pois observou uma maior prevalência de desnutrição grave nas mulheres, sendo de $41,18 \%$.

Dentre os fatores que podem contribuir para o bom estado nutricional da população é a ingestão alimentar, o nível sócio-econômico, as condições clínicas, o tempo e a frequência de diálise, a adequação dialítica e o tipo de membrana podem em menor ou maior grau influenciar nos parâmetros de avaliação nutricio$\mathrm{nal}^{24}$.

Segundo adequação da circunferência do braço podemos observar que os homens obtiveram um percentual maior de desnutrição leve. Esse fato é semelhante ao estudo dos autores ${ }^{13}$ que ao avaliarem 71 pacientes do sexo masculino submetidos a hemodiálise observaram que $88,1 \%$ destes apresentaram com desnutrição leve, $11,3 \%$ com eutrofia, 4,2 \% com sobrepeso e $1,4 \%$ com obesidade.

De acordo com a adequação da circunferência muscular do braço e da média da área muscular do braço corrigida, podemos observar que os pacientes do sexo masculino obtiveram uma média inferior à faixa de eutrofia, evidenciando maior perda de massa muscular nos homens.

Em um estudo ${ }^{22}$ a média de adequação da CMB obtida ao avaliarem 165 pacientes maiores de 18 anos de idade em hemodiálise, na Clínica Renal de Manaus, corrobora com a presente pesquisa, na qual os homens apresentaram média de $88,7 \% \pm 8,6$, evidenciando um grau de desnutrição leve. Esse fato se diferencia dos resultados encontrados por outros autores ${ }^{26}$ onde $66,67 \%$ dos pacientes avaliados de ambos os sexos apresentavam-se eutróficos, 22,22\% com excesso de peso e $11,11 \%$ com desnutrição grave.

Em relação à $\mathrm{AMBc}$, uma pesquisa anterior ${ }^{13}$ evidenciou que $81,7 \%$ dos pacientes do sexo masculino encontravam-se com desnutrição grave e 18,3\% com eutrofia, corroborando com os dados do presente estudo. Os resultados do presente estudo segundo $\mathrm{AMBc}$ demonstraram que a maioria dos pacientes do sexo masculino encontravam-se abaixo do percentil 5 indicando uma elevado grau de desnutrição energética proteica.
Os pacientes avaliados de ambos os sexos não apresentaram depleção de gordura corporal através da PCT, esse fato se diferencia do estudo ${ }^{25}$ cuja a perda de gordura corporal entre as mulheres foi quatro vezes mais frequente em relação aos homens.

A manutenção de uma reserva adequada de gordura corporal é indicada para o paciente em hemodiálise, pois em situações de maior demanda energética às quais o paciente está exposto como em casos de cirurgias de acesso vascular, infecções, ou até transplante renal a gordura reservada pode ser utilizada para suprir o déficit energético e poupar a utilização das reservas proteica ${ }^{27}$.

No que se diz respeito aos indicadores bioquímicos, estes são parte importante da avaliação nutricional dos pacientes com IRC, mas podem se alterar na presença dos vários distúrbios decorrentes da enfermidade e do processo de diálise. Assim esses marcadores devem ser interpretados com cautela e sempre em conjunto com demais parâmetros que fazem parte da avaliação nutricional ${ }^{23}$.

Constatou-se por meio dos resultados obtidos a partir da albumina que as mulheres apresentaram maior percentual de desnutrição leve, enquanto a maioria dos homens obtiveram valores adequados, diferenciando dos resultados de uma pesquisa ${ }^{13}$ na qual foram identificados níveis de albumina iguais ou maiores que $4,0 \mathrm{~g} / \mathrm{dL}$ em $60 \%$ dos paciente de ambos os sexos, sendo esses valores considerados normais.

Evidências ${ }^{28}$ mostram que o valor prognóstico e/ou diagnóstico da albumina sérica em pacientes submetidos à hemodiálise é atualmente o foco de discussões, já que as suas concentrações podem sofrer influências de uma gama de condições clínicas e nutricionais. Já está bem estabelecida que a hipoalbuminemia é um importante determinante da morbidade e mortalidade em pacientes com IRC.

Em relação aos outros indicadores bioquímicos do presente estudo, apenas os níveis séricos de ureia e cálcio em ambos os sexos não estavam dentro dos valores aceitáveis. Esses resultados diferenciam em parte do trabalho de outros autores ${ }^{26}$ que apresentou médias dos parâmetros bioquímicos gerais (cálcio, fósforo, potássio, ureia, creatinina) dentro do esperado para pacientes em hemodiálise.

Vários estudos demonstraram que baixas concentrações de ureia se associam a uma sobrevida reduzida, presumivelmente porque baixos níveis de ureia se refletem diminuição da massa muscular esquelética e uma baixa ingestão de proteínas, sendo assim um indicador de desnutrição ${ }^{29}$.

Estudo revela que a hipocalcemia na IRC não é frequente e decorre basicamente da má absorção 
intestinal pela carência de vitamina $\mathrm{D}$, do efeito quelante do fósforo, e do uso excessivo de diuréticos espoliadores de potássio ${ }^{30}$.

Os autores de uma pesquisa ${ }^{12}$ ao analisar a associação dos indicadores bioquímicos (uréia e creatinina) com o estado nutricional, considerando o IMC, não observou associações significativas ( $p>0,05)$, conforme observado no presente estudo.

\section{CONCLUSÕES}

Verificou-se no presente estudo que a maioria dos pacientes com IRC submetidos à hemodiálise é do sexo masculino, em idade entre 21 a 59 anos, com uma parcela maior daqueles que concluíram o ensino fundamental e dos que não concluíram o ensino médio, sendo a maioria com renda familiar de um salário mínimo.

Em se tratando da etiologia da IRC a Glomerulonefrite crônica e a Nefroesclerose Hipertensiva foram as doenças mais prevalentes em ambos os sexos.

A partir das medições antropométricas realizadas no presente estudo percebemos que a maioria das

\section{REFERÊNCIAS}

1. Cuparri L, Avesani, CM, Bufarah MNB, Baxmann AC. Doenças renais. In: Cuparri, L. Guia de Nutrição: Clínica do adulto. 3 a . ed. Barueri, São Paulo: Manoele; 2014.

2. Araujo IC, Kamimura MA, Draibe AS, Canziani MEF, Manfredi SR, Avesani, CM. Nutritional parameters and mortality in incident hemodialysis patients. J Renal Nutr. 2006; 16(1):27-35.

3. Pupim L, Cuppari L, Ikizler TA. Nutrition and metabolism in kidney disease. Seminars in Nephrology. 2006; 26(2):134-57.

4. Thomé FS, Gonçalves LF, Manfro RC, Barros E. Doença renal crônica. In: Barros E, Manfro RC, Thomé F, Gonçalves LF. Nefrologia: rotinas, diagnóstico e tratamento. 3a. ed. Porto Alegre: Artmed. 2007.

5. Shah SN, Abramowi TZM, Hostetter TH, Melamed ML. Serum bicarbonate levels and the progression of kidney disease: a Cohort Study. Am J Kidney Dis. 2009; 54(1):270-7.

6. Organização Mundial da Saúde. Obesity: preventing and managing the global epidemic. Report of a World Health Organization Consultation. WHO Obesity Technical Report. Series.284:256 Geneva: WHO, 2000.

7. Frisancho AR. Anthropometry standard for the assessment of growth and nutrition status. Michigan: The University of Michigan Press, 1993.

8. Blackburn GL, Thornton PA. Nutrition assessment of the hospitalized patients. Med Clin North Am. 1979; 63(1): 1103-1115. mulheres apresentaram-se eutróficas em todos os indicadores de medidas corporais utilizados, enquanto a maioria dos homens encontravam-se desnutridos de acordo com a $\mathrm{CB}, \mathrm{CMB}$ e $\mathrm{AMBc}$, revelando um déficit de massa muscular.

No que se refere aos exames laboratoriais observamos que a maioria das mulheres apresentou desnutrição leve de acordo com a albumina sérica. Em relação aos outros níveis séricos apenas a ureia e cálcio apresentaram valores não aceitáveis tanto para o sexo feminino como para o masculino, estando os valores dos outros indicadores bioquímicos dentro da faixa de normalidade.

Embora não tenha sido encontrada significância estatística entre as associações realizadas no presente estudo, observou-se que o acompanhamento e o monitoramento nutricional de rotina em pacientes com IRC são fundamentais para prevenção de distúrbios nutricionais comumente observados nesses pacientes. Como não existe um parâmetro que forneça de forma completa o estado nutricional, é necessário utilizar diversos indicadores em conjunto que permitam identificar riscos ou anormalidades nutricionais já instalados.

9. Riella MC, Martins C. Nutrição e hemodiálise. Nutrição e o rim. Rio de Janeiro: Guanabara Koogan, 2001.

10. Martins C. Protocolo de Cuidados Nutricionais. In: Riella MC, Martins C. Nutrição e o Rim. 1르 Ed. Curitiba: Guanabara Koogan, 2001.

11. Martins C, Cardoso SP. Terapia nutricional enteral e parenteral - Manual de rotina técnica, 2000.

12. Calado IL, França AKTC, Santos AM, Filho MS. Avaliação Nutricional de Pacientes Renais em Programa de Hemodiálise em um Hospital Universitário de São Luís do Maranhão. J Bras Nefrol. 2007; 29(4).

13. Silva RKB, Silva RKBB, Silva RPB. Perfil nutricional de pacientes renais crônicos da clínica Nefrológica de Caruaru-PE [trabalho de conclusão de curso]. Caruaru: Faculdade do Vale do Ipojuca, Curso de Nutrição; $2010.27 p$.

14. Mascarenhas CHM, Reis LA, Lyra JE, Peixoto AV, Teles MS. Insuficiência renal crônica: caracterização sóciodemográfica e de saúde de pacientes em tratamento hemodialítico no município de Jequié/BA. Revista Espaço para a Saúde. 2010; 12(1): 30-37.

15. Fausto MA, Gomes JG, Iglesias ACRG, Ferraz AS, Marchini JS. Marliere CA. Avaliação nutricional de pacientes com Insuficiência Renal Crônica submetidos à hemodiálise. Alim Nutr. 2006; 7(1): 15-23.

16. Pivatto $D R$, Abreu IS. Principais causas de hospitalização de pacientes em hemodiálise no município de Guarapuava, Paraná, Brasil. Rev Gaúcha Enferm. 2010; 31(3):515-20. 
17. Marcelino EHA. A percepção do paciente renal crônico em diálise peritoneal ambulatorial contínua quanto ao acompanhamento da equipe multiprofissional em um hospital de Dourados - MS [trabalho de conclusão de curso]. Dourados: Centro Universitário da Grande Dourados, Curso de enfermagem; 2008.

18. Madeiro AC, Machado PDLC, Bonfim IM, Braqueais AR, Francisca ETL. Adesão de portadores de insuficiência renal crônica ao tratamento de hemodiálise. Acta Paul Enferm. 2010; 23(4): 546-51.

19. Oliveira GTC. Avaliação nutricional de pacientes submetidos à hemodiálise em centros de diálise de Belo Horizonte [Dissertação] Belo Horizonte: Universidade Federal de Minas Gerais, Curso de Nutrição; 2010.145p.

20. Lanza AHB, Chave APA, Garcia, RCP, Brandão JA G Perfil biopsicossocial de pacientes renais crônicos em tratamento hemodialítico. Arquivos Brasileiros de Ciências da Saúde. 2008; 33(3):141-5.

21. Sociedade Brasileira De Nefrologia. Censo SBN 2013. Disponível em: http://www.sbn.org.br/. Acesso: 17 de junho de 2014

22. Valenzuela RGV, Giffoni AG, Cuppari L, Canziani MEF. Estado nutricional de pacientes com insuficiência renal crônica em hemodiálise no Amazonas. Rev Assoc Med Bras. 2003; 49(1): 72-8.

23. Cuparri L, Avesani CM, Kamimura M A. Nutrição na doença renal crônica. Barueri- SP: Manole, 2013.

24. Cardozo MT, Vieira IO, Campanella L C. A. Alterações nutricionais em pacientes renais crônicos em programa de hemodiálise. Rev Bras Nutr Clin. 2006; 21(4): 284-9.
25. Piratelli MC. Avaliação nutricional de pacientes em hemodiálise no município de Araraquara [Dissertação]. Araraquara: Universidade Estadual Paulista; 2009. 79p.

26. Marculino AQ. Avaliação nutricional do paciente submetido à hemodiálise [trabalho de conclusão de curso]. Criciúma: Universidade do Extremo Sul Catarinense - UNESC; 2004.84p

27. Kamimura MA, Draibe AS, Sigulem DM, Cuppari L. Métodos de avaliação da composição corporal em pacientes submetidos à hemodiálise. Rev. Nutr. 2004; 17(1).

28. Draibe AS, Santos NSJ, Kamimura MA, Cuppari L. Albumina sérica como marcador nutricional de pacientes em hemodiálise. Rev. Nutr. 2004; 17 (3):339-34.

29. Kopple JD, Massry SG. Cuidados nutricionais das doenças renais. Rio de Janeiro: Guanabara Koogan, 2006.

30. Helou, CMB. Potássio e Bicarbonato. J Bras Nefrol. 2004; 26(3).

\section{Correspondência}

Nome: Arabela Vieira Clementino

Endereço: Getúlio Vargas 193 Bairro: Centro

CEP: 58200-000

Cidade: Guarabira - Paraíba - Brasil

E-mail: arabela_vc@hotmail.com 